\title{
FLAT MODULES OVER COMMUTATIVE NOETHERIAN RINGS
}

\author{
BY \\ WOLMER V. VASCONCELOS
}

\begin{abstract}
In this work we study flat modules over commutative noetherian rings under two kinds of restriction: that the modules are either submodules of free modules or that they have finite rank. The first ones have nicely behaved annihilators: they are generated by idempotents. Among the various questions related to flat modules of finite rank, emphasis is placed on discussing conditions implying its finite generation, as for instance, (i) over a local ring, a flat module of constant rank is free, and (ii) a flat submodule of finite rank of a free module is finitely generated. The rank one flat modules already present special problems regarding its endomorphism ring; in a few cases it is proved that they are flat over the base ring. Finally, a special class of flat modules--unmixed-is discussed, which have, so to speak, its source of divisibility somewhat concentrated in the center of its endomorphism ring and thus resemble projective modules over flat epimorphic images of the base ring.
\end{abstract}

Introduction. This paper is an attempt to lay a somewhat limited framework for the study of flatness on modules over noetherian rings. As already for abelian groups the general description of the torsion-free modules is practically hopeless, we shall not be too demanding here and shall concentrate in those aspects of the theory which best reflect the nature of the ring and the universal algebra involved. In this vein, after some preliminaries on the rank of flat modules and a generalization of a theorem of [4] on how well the finite generation of a high nonzero exterior power of a module may disclose the finite generation of the module itself, we discuss: annihilators of flat submodules of free modules, which, in general, have a good behavior in comparison to annihilators of arbitrary flat modules; various conditions disguising the finite generation of a flat module; flat modules of rank one and its endomorphism rings - which are, in many cases, flat epimorphic images of the base ring; a discussion of a class of flat modules-unmixed modules-where the source of divisibility lies, in general, in the complement of an affine subschema of the spectrum of the ring. The paper concludes with a list of unsolved problems whose solutions might yield new methods or, more hopefully, uncover other avenues of interest.

1. Preliminaries. Throughout, $R$ will denote a commutative ring with unity. If $R$ is a domain with field of quotients $K$ and $M$ is an $R$-module, we recall that the

Received by the editors January 28, 1970.

AMS subject classifications. Primary 1340; Secondary 1325.

Key words and phrases. Flat modules, integrally closed noetherian domains, flat overrings, unmixed modules.

Copyright (C) 1970, American Mathematical Society 
rank of $M$ is the dimension of $M \otimes K$ as a vector space over $K$, i.e. the rank of $M$ is the maximum rank of free submodules of $M$. For a general $R$ we shall use

Definition 1.1. The rank of $M$ at a prime ideal $P$ is the rank of $M / P . M$ as an $R / P$-module. It will be denoted by $\operatorname{rk}_{M}(P)$.

Another notion of rank is obtained by the use of exterior powers [3]. For an $R$-module $M$

Definition 1.2. The exterior rank of $M$ is the order of the highest nonzero exterior power of $M$. It will be denoted by ext $\mathrm{rk} M$.

This rank is a good indication of the number of elements necessary to generate $M$ and is related to (1.1) in the following manner: $\operatorname{rk}_{M}(P) \leqq \operatorname{ext} \mathrm{rk} M / P . M$ where equality seldom holds. This follows because for a domain the rank of a module is the order of the highest exterior power of the module which is not a torsion module. In the case of flat modules however, one always has equality.

To simplify some of the later arguments we need the following which generalizes a theorem of Flanders [4].

TheOREM 1.3. Let $R$ be a commutative ring and $M$ an $R$-module. Suppose that for some positive $n, \bigwedge^{n} M$ is a projective module of rank one. Then $M$ is a finitely generated projective module of rank $n$.

Proof. By [9] one knows that $P=\bigwedge^{n} M$ is a finitely generated $R$-module, generated by, say, $m_{1}^{(i)} \wedge \cdots \wedge m_{n}^{(i)}, 1 \leqq i \leqq p$. We shall prove that at each localization one of the sets of elements $m_{1}^{(i)}, \ldots, m_{n}^{(i)}$ generates $M$; by the globalization principle $M$ will then be finitely generated. As localization and formation of exterior powers commute, assume $R$ local; we may then elect one of the $n$-vectors above, say $m_{1} \wedge \cdots \wedge m_{n}$, as the generator of $P$. Now the $m_{i}$ 's generate a free submodule of $M$ as any relation $\sum r_{j} m_{j}=0$ by multiplication by $m_{1} \wedge \cdots \wedge\left(m_{k}\right)^{\wedge} \wedge \cdots \wedge m_{n}$ leads to $\left( \pm r_{j}\right) m_{1} \wedge \cdots \wedge m_{n}=0$ and thus $r_{j}=0$. Write $F$ for the submodule they generate, and let

$$
S=\left\{m \in M \mid m \wedge m_{1} \wedge \cdots \wedge\left(m_{k}\right)^{\wedge} \wedge \cdots \wedge m_{n}=0,1 \leqq k \leqq n\right\} .
$$

$S$ is a submodule of $M$ and $F \cap S=(0)$. Further, if $m \in M$, let $m^{\prime}=\sum r_{k} m_{k}$ where $r_{k}$ is the coefficient of $m_{1} \wedge \cdots \wedge m_{k-1} \wedge m \wedge m_{k+1} \wedge \cdots \wedge m_{n}$ as a multiple of $m_{1} \wedge \cdots \wedge m_{n}$. Then $m-m^{\prime} \in S$, thus showing that $M=F \oplus S$. Taking exterior powers of order $n$, one gets

$$
R \cong \bigwedge^{n} M=\bigwedge^{n} F \oplus\left(\bigwedge^{n-1} F\right) \otimes S \oplus \cdots
$$

from which it follows $S=(0)$. This shows that $M$ is a finitely generated flat module of constant rank and it then follows (e.g. [8]) that it is projective.

2. The annihilator of a flat module. Annihilators of arbitrary flat modules can be wild as for any nondomain $R$ "most" localizations $R \rightarrow R_{S}$ ([5] has several examples) have nontrivial kernels, which become the annihilators of the $R_{S}$ 's. If, however, $R$ is noetherian and $M$ is a flat submodule of a projective module $F$, the situation is much more pleasant. 
THEOREM 2.1. (i) The annihilator of $M$ is generated by an idempotent $e$.

(ii) The support of $M$ is the open-closed set $V(e)(V(I)=\{P=$ prime ideal, $P \supseteq I\})$.

Proof. (i) Write $I=$ annihilator $M=\operatorname{Ann}(M)$; for $a \in I, m \in M$, $a m=0$ and by the flatness of $M$ one has $m=\sum r_{i} m_{i}, a r_{i}=0$. This shows $m \in \operatorname{Ann}(a) . M$. If $a_{1}, \ldots, a_{n}$ is a generating set for $I$, one has

$$
M=\operatorname{Ann}\left(a_{1}\right) \cdot M \cap \cdots \cap \operatorname{Ann}\left(a_{n}\right) 1 M=\left(\operatorname{Ann}\left(a_{1}\right) \cap \cdots \cap \operatorname{Ann}\left(a_{n}\right)\right) \cdot M
$$

with the last equality again by the flatness of $M$. Thus $M=J . M, J=$ annihilator $I$. As $M$ is a submodule of a projective module $F, M \subseteq \bigcup\left(J^{n} . F\right)=\left(\bigcup J^{n}\right) . F$. By the Krull intersection theorem [10], there is $a \in \bigcap J^{n}$ such that $(1+a) \cdot\left(\bigcap J^{n}\right)=(0)$ and thus $1+a \in I$. This shows $I+J=R$ and $I \oplus J=R$ follows. We can thus write $I=R e, e=e^{2}$.

(ii) Let $P$ be a prime ideal. If $M=P . M$, as in (i) one gets $(1+p) M=(0)$ for $p \in P$ and thus $M_{P}=(0)$. It follows easily then that $M_{P}=(0)$ if and only if $P \supseteq J$. Thus Supp $M=V(e)$.

Corollary 2.2. If $M$ is a flat submodule of a free module, then $\left\{P \mid \operatorname{rk}_{M}(P) \geqq n\right\}$ is an open-closed subset of Spec $R$.

Proof. It follows from [5] that any inclusion $M \subseteq P$ of flat modules extends to an inclusion of their exterior powers; now use (2.1).

COROLlaRy 2.3. If $M$ is a flat submodule of a free module, with finite rank at each prime, then $M$ has finite exterior rank.

Proof. Follows from (2.2) and the fact that $R$ does not admit a strictly decreasing sequence of idempotent ideals.

REMARK 2.4. If $R$ is not noetherian (2.1) fails to hold already in the following case: $R=$ ring of all real continuous functions defined on the unit interval and $M=$ ideal of functions null in a neighborhood of 0 . Annihilator $M=0, M$ is projective but not faithfully flat.

3. Finite generation of flat modules. In [2, p. 23] one finds the abelian group $M=\bigcup Z d^{-1}, d=$ square-free, which is at each prime isomorphic to $Z$ but not finitely generated. This and other simple examples of abelian groups suggest that very strict conditions must be placed on a flat module to ensure its finite generation. In this section we shall discuss a few of those disguises. Throughout $R$ will denote a noetherian ring.

THEOREM 3.1. A flat module $M$ of constant rank over a local ring $R$ is finitely generated (and thus free).

Proof. Let $M$ be a flat module of rank $n$. To use (1.3) consider $\wedge^{n} M$. We shall prove that $\bigwedge^{n} M \simeq R$. Change notation and assume that $M$ has rank one. We have $M / P . M \simeq R / P$, where $P$ is the maximal ideal of $R$; let $m$ be an element of $M-P . M$. We prove by induction on the Krull dimension of $R$ that $m$ generates $M$. 
Also, we can "mod out" the nil radical of $R$ without changing anything; finally, assume that for any nonminimal prime $Q, m$ generates $M / Q . M$ over $R / Q$. Let $N=R m$ and consider the exact sequence

$$
0 \rightarrow N \rightarrow M \rightarrow C \rightarrow 0 .
$$

Pick $d \in R$ which is not a zero divisor, that is, $d$ is not in any minimal prime. Then the kernel-cokernel sequence induced by multiplication on $\left({ }^{*}\right)$ yields

$$
0 \rightarrow{ }_{d} C \rightarrow N / d N \rightarrow M / d M \rightarrow C / d C \rightarrow 0
$$

where ${ }_{d} C$ denotes the elements of $C$ annihilated by $d$. By the induction hypothesis $M / d M \simeq R /(d)$, generated by the image of $m$. Thus $M / d M \simeq N / d N$ and ${ }_{d} C=0$, $C=d C$. This says that $C$ is naturally a module over $K$, the total ring of quotients of $R$ (which is semisimple) and is thus a flat module. It follows that $N$ is also a flat module and is thus free. Now tensor the exact sequence $\left(^{*}\right)$ by $K$ to get

$$
0 \rightarrow N \otimes K \rightarrow M \otimes K \rightarrow C \otimes K \rightarrow 0 \text { and } C=(0) .
$$

In case $R$ is no longer local but $M$ is a submodule of a projective module one has

THEOREM 3.2. If $M$ is of finite rank at each prime, then $M$ is finitely generated.

Proof. By (2.2) and suitable choice of idempotents one can split $R=\oplus \sum \boldsymbol{R e}_{\boldsymbol{i}}$ and $M=\oplus \sum e_{i} M$, where $e_{i} M$ has constant rank over $R e_{i}$. Change notation and assume that $M$ is of constant rank over $R$. Let $K$ be its total ring of quotients. As $M$ is a submodule of a free module, to show $M$ finitely generated it is enough to prove that $M$ has nonzero coordinates in only finitely many basic components of the free module. This will be shown if $M \otimes K$ is proved finitely generated; as $K$ is semilocal, the result now follows from (3.1).

4. Flat modules of rank one. For a flat $R$-module $M$ let $\Omega=\operatorname{End}_{R}(M)$ and let $S$ be the center of $\Omega$, i.e. $S=\operatorname{End}_{\Omega}(M)$. Questions concerning the possible flatness of $\Omega$ are very elusive and we shall be contented with examining the flatness of $S$ in some cases.

We begin with a result which permits the comparison of submodules of a special kind by looking at their localizations at the minimal primes. We recall a generalization of the notion of associated prime ideal of a module.

If $P$, a prime ideal, is minimal over Annihilator $R m, m \in M$, then $P$ is said to be weakly associated to $M$. (If $R$ is noetherian the two notions coincide.) For the moment, we shall assume that $R$ is an arbitrary domain.

Lemma 4.1. A prime ideal $P$ minimal over $(a): b$ is of grade one.

Proof. Assume $x, y$ is an $R$-sequence in $P$. It is immediate that $x^{n}$ and $y^{n}$ is also an $R$-sequence. As $P$ is minimal over $(a): b$, by localization, say, it follows that for some exponent $n$ and some element $s \notin P, s x^{n}, s y^{n} \in(a): b$. Thus $b s x^{n}=c a$ and $b s y^{n}=d a$; from $b d s x^{n}=b c s y^{n}$ we have $d x^{n}=c y^{n}$. Thus $d=e y^{n}$ and $s b=e a$, a contradiction as $s \notin P \supseteq(a): b$. 
We shall denote by $W$ the set of all prime ideals weakly associated to modules like $R /(a)$. By (4.1) they all have grade one and in case $R$ is noetherian it can be easily proved that $P \in W$ if and only if grade $P R_{P}=1$.

Proposition 4.2. Let $M$ be a torsion-free module over a domain $R$. If $M=$ $\bigcap_{P \in W} M_{P}$ then every $R$-sequence of two elements is also an $M$-sequence. The converse holds if $R$ satisfies the following condition: Every ideal I not contained in any $P \in W$ has grade greater than one (e.g. $R$ noetherian or a Krull domain).

Proof. Let $M$ be as above and let $a, b$ be an $R$-sequence. If $a m=b n$, we must show $m \in b M$. $M^{\prime}=b M$, being isomorphic to $M$, can also be represented as $M^{\prime}=\bigcap_{P \in W} M_{P}^{\prime}$. Let $P \in W$; then by (4.1) either $a$ or $b$ is not in $P$ and it follows $m \in M_{P}^{\prime}$.

For the converse consider $m \in \bigcap_{P \in W} M_{P}$. Let $I=\{r \in R \mid r m \in M\}$; by construction $I$ lies outside of any $P \in W$ and so contains an $R$-sequence $a, b$. But $b(a m)=$ $a(b m)$ implies $a m=a m^{\prime}, m^{\prime} \in M$, thus proving $m \in M$.

Corollary 4.3. Let $M$ be a flat module over a noetherian domain $R$. Then $M=\bigcap_{P \in W} M_{P}$.

If $R$ is a domain, with field of quotients $K$, one first observation to be made about the center $S$ of the endomorphism ring $\operatorname{Hom}_{R}(M, M)$ of a flat module $M$ of rank $n$ is

Proposition 4.4. $S$ can be identified to a subring of $K$.

Proof. Let $S^{\prime}=\operatorname{End}_{R}\left(\bigwedge^{n} M\right)$, which is naturally a subring of $K$. We define the following map $\omega: S \rightarrow S^{\prime}:$ for $f \in S$, let $\omega(f)=f \wedge I_{M} \wedge \cdots \wedge I_{M} ; \omega$ is clearly a $R$-homomorphism of rings. If $f \neq 0$, let $m$ be such that $f(m) \neq 0$ and pick $m_{2}, \ldots, m_{n}$ elements in $M$ such that $f(m), m_{2}, \ldots, m_{n}$ is a linearly independent set. That this choice can be made implies $\omega(f) \neq 0$.

If $R$ is now a noetherian domain in which principal ideals are height unmixed, then the endomorphism ring of a rank one flat module behaves well under localization.

If $T$ is a multiplicative set in $R$ and $M$ is the flat module in question, denote $M \otimes R_{T}=M_{T}$. We then have

Proposition 4.5. $\operatorname{Hom}_{R}(M, M)_{T}=\operatorname{Hom}_{R_{T}}\left(M_{T}, M_{T}\right)$.

Proof. When $M$ is identified to a submodule of $K$, its endomorphism ring $S$ is just $M: M=\{x \in K \mid x M \subseteq M\}$. Because every $R$-sequence of 2 elements is also an $M$-sequence, it follows easily that $\operatorname{Hom}_{R}(M, M)$ satisfies the same property. Write $S^{T}=M_{T}: M_{T}$. Now $S_{T} \subseteq S^{T}$ and both submodules of $K$ satisfy the condition on the $R$-sequences of length 2 . In applying (4.2) it is enough to use prime ideals in $W$ which avoid $T$. Let $P$ be one such; if $P . M \neq M$, then $M_{P} \cong R_{P}$ as in (3.1). In this case $\left(S_{T}\right)_{P} \subseteq\left(S^{T}\right)_{P} \subseteq R_{P} \subseteq\left(S_{T}\right)_{P}$. Assume then that $P . M=M$; in this case 
$\left(P^{n}\right)^{-1} \subseteq S$ for all integers $n$ and thus $S_{P} \supseteq \bigcup\left(P^{n}\right)_{P}^{-1}=K$ and again $\left(S_{T}\right)_{P}=\left(S^{T}\right)_{P}$. In case $R$ is a noetherian domain in which the primes in $W$ are invertible (i.e. if $R$ is also locally a UFD) the ring $S$ above is much more explicitly described.

THEOREM 4.6. $S$ is a flat epimorphic image of $R$ (i.e. $R \subseteq S \subseteq K$ and $S$ is $R$-flat).

Proof. In order to see this we use the Akiba-Richman [1], [6] characterization of flat epimorphisms. Let $Q$ be a prime ideal in $S$ and $P=Q \cap R$. By the previous result, $S$ localizes and thus we can assume $R$ local, with maximal ideal $P$ and $P S \neq S$. We claim $S=R$. Assume they are unequal at some prime $V$ of height one; then $S_{V}=K$. This leads to $V . M=M$ or $V^{-1} \cdot M=M$ and $V^{-1} \subseteq S$. Thus $1 \in P . V^{-1}$ and so $P S=S$, a contradiction.

It should be interesting to know whether the same holds for a wider class of rings.

5. Unmixed modules. For simplicity we assume that $R$ is a domain.

Definition 5.1. A flat $R$-module $M$ of rank $n$ is said to be unmixed if for each prime ideal $P, \operatorname{rk}_{M}(P)=0$ or $n$.

This means that either $M=P . M$ or that $M / P . M$ has rank $n$ as an $R / P$-module. If $R$ is assumed noetherian it can be seen by a combination of (2.1) and (3.1) that this last case is equivalent to $M_{P}$ being a free $R_{P}$-module. One could also refine this definition by restricting the set of primes as in the following.

Proposition 5.2. Let $R$ be a noetherian domain which is locally $a$ UFD and $M$ a flat module of rank $n$. If for each prime ideal $P$ of height one either $M=P . M$ or $M_{P}$ is $R_{P}$-free of rank $n$, then $S$, the center of $\operatorname{End}_{R}(M)$, is a flat overring of $R$.

Proof. Let $N=\bigwedge^{n} M$. By (4.6), $S^{\prime}=\operatorname{End}_{R}(N)$ is a flat overring of $R$ and the claim is that the homomorphism of (4.4) is an isomorphism. For that it will be sufficient to show that $M=S^{\prime} . M=S^{\prime} \otimes M$. Since $M$ is flat, we can apply (4.2). Let $P$ be a prime of height 1 ; if $P . M=M$ then $M_{P} \cong K^{n}$ and there is nothing to worry about. If $M_{P} \cong R_{P}^{n}, S_{P}^{\prime}=R_{P}$.

This notion of unmixedness seems to convey the idea that a flat module so endowed has its divisibility somewhat concentrated, as in the case above, in an affine subschema of Spec $R$.

We conclude with the following generalization of a piece of a theorem of Silver [7], where unmixed (then unnamed) modules first appeared.

THEOREM 5.3. Let $M$ be a torsion-free module of rank $n$ over a noetherian domain with unmixed (by height) principal ideals. Then the following are equivalent:

(i) There exists a flat overring $S$ of $R$ such that $M$ is naturally a projective $S$ module.

(ii) $M$ is $R$-flat and a projective $\operatorname{End}_{R}(M)$-module.

The proof can be traced to the preceding techniques, specially (4.5), plus the Morita equivalence. 


\section{Some open problems.}

Problem 6.1. Let $M$ be a flat module over the commutative ring $R$. Call $M$ algebraic if $\operatorname{End}_{R}(M)$ is integral over $R$. The conjecture is, if $M$ is algebraic then $M$ is of finite rank (at least when $R$ is noetherian).

Problem 6.2. Is an epimorphism of a flat module of finite rank necessarily an isomorphism?

Problem 6.3. Let $R$ be a noetherian ring and $M$ a flat module of finite rank. Is $\operatorname{End}_{R}(M)$, or at least its center, $R$-flat?

Finally, more in the nature of an exercise,

Problem 6.4. Let $\alpha$ be an $n \times n$ matrix with coefficients in the commutative ring $R$. Consider the directed system

$$
\longrightarrow R^{n} \stackrel{\alpha}{\longrightarrow} R^{n} \stackrel{\alpha}{\longrightarrow} R^{n} \longrightarrow
$$

and let $M$ be its limit. We conjecture that $M$ is finitely generated and projective if and only if each Fitting's invariant $I$ of $\alpha$ is asymptotically idempotent, i.e. for large $m, I^{m}=I^{m+1}$.

\section{REFERENCES}

1. T. Akiba, Remarks on generalized rings of quotients, Proc. Japan Acad. 40 (1964), 801-806. MR 31 \#4807.

2. H. Bass, K-theory and stable algebra, Inst. Hautes Études Sci. Publ. Math. 22 (1964), 5-60. MR 30 \#4805.

3. C. Chevalley, Fundamental concepts of algebra, Academic Press, New York, 1956. MR 18, 553.

4. H. Flanders, On free exterior powers, Trans. Amer. Math. Soc. 146 (1969), 360-367.

5. D. Lazard, Autour de la platitude, Bull. Soc. Math. France 97 (1968), 81-128.

6. F. Richman, Generalized quotient rings, Proc. Amer. Math. Soc. 16 (1965), 794-799. MR 31 \#5880.

7. L. Silver, personal communication.

8. W. V. Vasconcelos, On finitely generated flat modules, Trans. Amer. Math. Soc. 138 (1969), 505-512. MR 39 \#199.

9. - On projective modules of finite rank, Proc. Amer. Math. Soc. 22 (1969), 430-433.

10. O. Zariski and P. Samuel, Commutative algebra. Vol. I, The University Series in Higher Math., Van Nostrand, Princeton, N. J., 1958. MR 19, 833.

RUTGERS UNIVERSITY,

New Brunswick, New Jersey 08903 